\title{
GRAVITATIONAL INSTABILITY ON PROPAGATION OF MHD WAVES IN ASTROPHYSICAL PLASMA
}

\section{Alemayehu Mengesha Cherkos1,2 AND S. B. Tessema 1,2}

1. Entoto Astronomical Observatory and Space Science Research Center, P.O. Box 8412, Addis Ababa, Ethiopia

2. Department of Physics, Kotebe College, P.O. Box 31248, Addis Ababa, Ethiopia (alexye9@yahoo.com)

\section{Abstract}

We determine the general dispersion relation for the propagation of magnetohydrodynamic (MHD) waves in astrophysical plasma by considering the effect of gravitational instability and viscosity with anisotropic pressure tensor and heat-conducting plasma. Basic MHD equations have been derived and linearized by the method of perturbation to develop the general form of dispersion relation equation. Our result indicates that the transverse propagation of waves in such a plasma is affected by the inclusion of heat conduction. For wave propagation, parallel to the magnetic field direction, we find that the fairhose mode is unaffected, whereas the mode corresponding to the gravitational instability is modified in astrophysical plasma with anisotropic pressure tensor being stable in the presence of viscosity and strong magnetic field at considerable wavelength. 